\title{
Protein Microarrays: From Serodiagnosis to Whole Proteome Scale Analysis of the Immune Response Against Pathogenic Microorganisms
}

\author{
Tito Bacarese-Hamilton ${ }^{1}$, Francesco Bistoni ${ }^{2}$, and Andrea Crisanti ${ }^{1}$ \\ ${ }^{1}$ Department of Biology, Imperial College of Science, Technology and Medicine, London, UK, and \\ ${ }^{2}$ Dipartimento di Medicina Sperimentale e Scienze Biochimiche, Universita' degli Studi di Perugia, Perugia, Italy
}

BioTechniques 33:S24-S29 (December 2002)

\section{ABSTRACT}

Microbial diseases remain the most common cause of global mortality and morbidity. Scientific and technical achievements have dramatically improved the possibilities of investigating the humoral immune response against the whole proteome of microbial organisms. A number of genomes of microbial organisms responsible for diseases of worldwide medical importance such as Plasmodium, Toxoplasma, Mycobacterium, Streptococcus, Neisseria, Salmonella, Borrelia, and Rickettsia species have already been sequenced or will be available in the very near future. High-throughput assays such as protein microarrays have been clinically validated in serum for detecting the presence of antibodies directed against microbial antigens. Computational technologies for processing large sets of data are rapidly being developed. Such a powerful combination of genomic information and assays now offers the opportunity to identify the microbial antigens that, either alone or in combination, function as targets of natural acquired immunity against infectious diseases. This information will prove invaluable for developing vaccines against a series of microorganisms of medical relevance that are urgently needed, e.g., malaria. Additional applications of these technologies include the development of a microbial antigen array for the early serodiagnosis of both common and rare infectious diseases.

This review will focus on technical and scientific issues concerning the use of antigen microarrays for vaccine development and the serodiagnosis of infectious diseases.

\section{INTRODUCTION}

Studies and epidemiological observations have shown that both natural and experimentally induced immunity against bacterial and parasite molecules protect against subsequent infection, thus providing the rationale for developing novel and effective vaccines against a series of devastating diseases that have, so far, been difficult to control. For example, in the case of malaria, for which a vaccine is urgently needed, people living in endemic countries who are continuously exposed to Plasmodium falciparum slowly develop a form of natural acquired immu- nity (NAI) that significantly reduces malaria morbidity and the rate of severe disease episodes (2). Progress in understanding how the immune response ultimately controls the infectivity and the growth of the malaria parasite has been slow because of the lack of suitable animal models and in vitro assays for measuring the level of protective immunity in vivo. None of the immune responses identified in humans against $P$. falciparum antigens correlates unequivocally with either protection from infection or decreased morbidity. This lack of basic knowledge has hampered the development of an effective vaccine $(4,13,27)$.

Studies of vaccine development and immune response have focused on analyzing either the entire organism (killed or attenuated) or a few antigens at a time. Bacterial- and parasite-encoded molecules have been studied and proposed as vaccine candidates with a priority that has been largely dominated by the stochastic order in which the corresponding genes have been discovered. Now the genome sequences of many bacteria and parasites of great medical importance are nearly completed. This provides about 4000-5000 candidate proteins for each microorganism and multiple variants of many of the corresponding genes. New experimental approaches must be sought to identify, among this challenging number of microbial molecules, those that are responsible for inducing the NAI observed in the field. DNA microarrays have been utilized to unravel genes differentially expressed by microorganisms under different growth conditions and at different developmental stages, as well as to identify genes selectively expressed by virulent strains $(3,16)$. This has significantly increased our knowledge of environmental- and stage-specific transcripts in different microorganisms $(20,30)$. Proteomic analysis and developments in liquid chromatography mass spectrometry promise to unravel the entire repertoire of proteins expressed at different metabolic and developmental stages in distinct subcellular compartments (8). Genomic information, transcription profiles, and proteomic analysis will be extremely helpful in predicting protein function and their likely subcellular localization, as well as to identify genes that contribute to virulence and pathogenesis. However, all these data 
Table 1. Comparison of Microarray and ELISA for Serum Reactivity to Allergens

\begin{tabular}{|c|c|c|c|c|c|c|}
\hline \multirow[b]{2}{*}{ Allergens } & \multicolumn{2}{|c|}{ ELISA } & \multicolumn{4}{|c|}{ Microarray } \\
\hline & Positive & Negative & Positive & Negative & Sensitivity \% & Specificity \% \\
\hline $\begin{array}{l}\text { Dermatophagoides } \\
\text { pteronyssinus }\end{array}$ & $11 / 22$ & $11 / 22$ & $13 / 22$ & $9 / 22$ & 90.9 & 72.7 \\
\hline D. farinae & $8 / 16$ & $8 / 16$ & $9 / 16$ & $7 / 16$ & 87.5 & 75 \\
\hline Olea europaea & $7 / 22$ & $15 / 22$ & $8 / 22$ & $14 / 22$ & 71.4 & 80 \\
\hline Alternaria alternata & $3 / 20$ & $17 / 20$ & $4 / 20$ & $16 / 20$ & 100 & 94.1 \\
\hline Artemisia vulgaris & $2 / 22$ & $20 / 22$ & $2 / 22$ & $20 / 22$ & 50 & 95 \\
\hline Dactylis glomerata & $6 / 22$ & $16 / 22$ & $7 / 22$ & $15 / 22$ & 83.3 & 87.5 \\
\hline
\end{tabular}

combined together will provide little information about the ability of microbial molecules to elicit a protective immune response. High-throughput assays for measuring the cell-mediated and humoral immune responses against a vast repertoire of microbial proteins in large groups of individuals would be needed to fill the gap between genomic data and vaccine development.

Available protein microarray technology represents a suitable tool to investigate the humoral immune response against the entire proteome of microbial organisms. Arrayed proteins retain their ability to interact specifically with other proteins, antibodies, or with small molecules in solution. Proteins chips have been employed to screen biochemical activities, protein-protein, protein-DNA, and protein-ligand interactions in a highthroughput fashion (22) and to reveal the presence of low concentrations of multiple analytes, such as cytokines and hormones and tumor antigens in complex solutions $(22,39)$. The entire proteome of a yeast has been arrayed on glass slides and screened for binding of proteins and lipids (38). The past 2 years have also seen remarkable progress in the development of antigen microarrays for the detection of specific antibodies in clinical samples $(1,18,24,31)$. These findings demonstrate the enormous potential of protein microarrays for studying the humoral immune response of large groups of individuals against bacterial and parasitic proteins on a whole-genome scale. Challenges that need to be overcome include: $(i)$ the production and the array of a large number of recombinant microbial proteins; (ii) the validation, in terms of performance and stability, of each arrayed antigen; and (iii) the development of novel array formats for studying the cell-mediated immune response.

\section{PROTEIN MICROARRAY TECHNOLOGY}

More than a decade ago, the ambient analyte assay theory described the principle of miniaturized multiple microspot ligandbinding assay $(10,11)$. It was demonstrated that an assay format using a small amount of capture molecule and sample could be extremely sensitive. This is due to the combination of two factors that improve both the detection and the kinetics of capture molecule-sample ligand interactions. The capture molecules are packed in a small area, and this maximizes the signal density generated by the ligand. The capture molecules subtract only minute amounts of ligand from the sample, and therefore, its concentration does not decrease over time. By using this system, thyroid-stimulating hormone (TSH) and hepatitis B surface antigen (HbsAg) could be quantified down to femtomolar concentrations $(10,11)$. The establishment of automated robust microdeposition technologies has recently allowed the development of high-density ordered arrays of proteins and nucleic acids (29). This assay format incorporates key features, such as true parallelism, miniaturization, and highthroughput, that could overcome most of the current enzyme-linked immunosorbent assay (ELISA) limitations
Figure 1. Schematic representation of the array employed for the clinical diagnosis of infectious diseases. Colored circles indicate where the antigen preparations were printed in replicate. The array contains internal calibration curves generated by printing increasing amounts of IgG and IgM, with rabbit myosin as the negative control. The fluorophores Alexa ${ }^{\mathrm{TM}} 546$ and Alexa 594 (Molecular Probes, Eugene, OR, USA) were also included in the array. 
(35), which include the requirement of large quantities of both sample and reagents, limited throughput, and time-consuming procedures, thus ultimately limiting their application for mass screening $(19,32)$. Ordered arrays of peptides have been used for unraveling protein-protein and protein-nucleic acid interactions $(7,12)$. Previous reports have shown that protein arrays printed on membranes can be utilized to screen expression libraries for binding to target molecules, as well as to unravel protein binding to either RNA or DNA $(5,6,14,21)$. More recently, arrays have been generated for high-throughput screening of recombinant antibodies (9). These antibody arrays contain thousands of bacterial clones, each expressing a different single chain antibody. Proteins covalently bound to glass slides were shown to retain their ability to interact specifically with other proteins or with small molecules in solution (22). Protein arrays have also been used to develop comparative fluorescence assays to measure the concentrations of several specific proteins and antibodies in complex solutions $(15,17,36,37)$. These studies have shown that protein microarrays could provide a practical means to quantify thousands of different protein species in clinical or research applications. In spite of these advances, the development of protein arrays for research and clinical applications has lagged behind, because of the poor stability of proteins, complex coupling chemistry, and weak detection signals. High-density protein arrays remain difficult to generate and validate for clinical use.

\section{PROTEIN MICROARRAYS FOR CLINICAL DIAGNOSIS}

Protein arrays were initially employed for diagnostic immunoassays, in which several sample parameters could be simultaneously analyzed. In the simplest configuration, utilized for serodiagnosis, arrayed antigens are exposed to clinical samples. Bound immunoglobulins (Ig) are revealed using labeled secondary antibodies. Protein microarrays have been used to assess the titer of autoantibodies directed against a series of diagnostic markers for autoimmune diseases arrayed on a glass slide (18). Protein microarrays have also been employed for determining in human sera the presence of specific IgG and IgM antibodies directed against parasitic and viral antigens including Toxoplasma gondii, cytomegalovirus, rubella virus, and herpes simplex virus types 1 and 2 (Figure 1). Regression analysis demonstrated that linear dose-response curves are obtained by plotting photomultiplier counts against bound Igs (Figure 2). These microarray assays incorporated internal calibration curves for each Ig subclass, which provides unique advantages over current immunoassay protocols, where the standard curve and the sample are processed in separate tubes or wells. In conventional assays, matrix problems (differences between the standard and sample matrix) occur frequently and represent a known source of bias. In contrast, in microarray assays the internal dose-response curve compensates for variability in the physicochemical conditions of the sample, thus minimizing the occurrence of matrix effects (24). These studies demonstrated that a protein microarray assay, using indirect fluorescence, can be employed to determine in human sera the presence or absence of specific antibodies directed against a variety of antigens (Figure 3 ).

The analytical sensitivity of the assays was around $0.5 \mathrm{pg}$ of antibody bound to specific antigens, which is well within the range of performance of clinically validated ELISA kits (24). Higher levels of sensitivity require the introduction of an amplification step in the detection procedure. Two different amplification protocols have been developed to reveal allergen-specific $\mathrm{IgE}$, which circulate in the serum at subnanomolar concentrations. The first protocol utilizes an adaptation of the rolling circle amplification (RCA) reporter system for the detection of bound antibodies called immunoRCA. Here an oligonucleotide is covalently attached to a secondary antibody. In the presence of circular DNA, DNA polymerase, and nucleotides, amplification results in a long DNA molecule containing hundreds of copies of

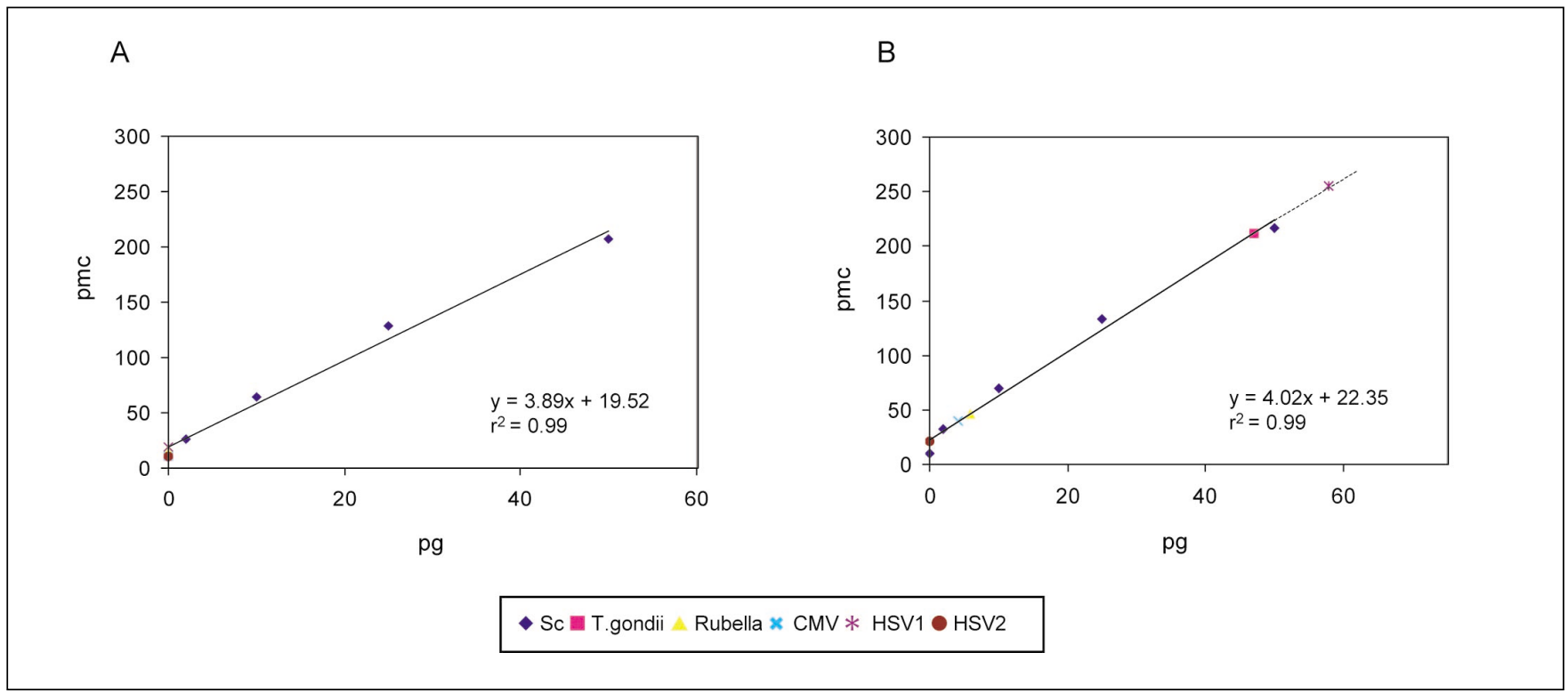

Figure 2. Dose-response curves generated by arrays incubated with serum samples. The arrays were incubated with two different serum samples (A and B), and measurements were interpolated from the dose-response curves generated by plotting the fluorescence intensity as a function of printed amounts of IgG (blue diamonds). 
the circular DNA sequence that remain attached to the antibody and that can be detected in a variety of ways (31). Using immunoRCA, analytes were detected at sensitivities exceeding those of conventional enzyme immunoassays. Microarrays incorporating a RCA amplification procedure have been used in clinical studies to detect allergen-specific IgE. The results validated the use of the RCA amplification step in microarray immunoassays for the detection of allergen-specific IgE with a performance similar to commercially available assays (31). The second protocol utilizes tyramide signal amplification to develop a high sensitivity microarray assay to reveal the presence of allergen-specific IgE in human sera. Tyramide signal amplification uses the catalytic activity of horseradish peroxidase (HRP) to generate high-density labeling of a target protein or nucleic acid sequence in situ (26). This procedure has been previously used for nucleic acid detection (25) or immunohistochemistry (33). The results of this work showed that the tyramide amplification system, similarly to RCA, dramatically increased the performance of protein microarray assays. Using $10 \mu \mathrm{L}$ of serum, $<1 \mathrm{fg}$ of bound antibody could be easily detected (1) (Figure 4). Moreover, a clinical study performed using an allergen microarray incorporating tyramide amplification showed good concordance with commercial ELISAs (Table 1). These studies demonstrate the enormous potential of protein microarrays in the serodiagnosis of infectious diseases and for studying the immune response of large groups of individuals against bacterial and parasitic proteins.

\section{ANALYSIS OF THE IMMUNE RESPONSE AGAINST MICROORGANISMS ON A WHOLE-GENOME SCALE}

Protein microarrays represent the tool of choice for studying the humoral immune response against the entire protein repertoire of complex microorganisms, such as bacteria and parasites. Available technology can be used to array on a glass slide all 4000-6000 proteins usually encoded by bacterial and parasitic genomes. These arrays could prove invaluable in unraveling the complexity of the humoral immune response against a vast repertoire of microbial-encoded molecules and to identify the antigens that, either alone or in combination, function as a target of the protective immunity. The major limiting step for the development of microrrays, containing the entire parasitic and bacterial proteome, is the production and purification of a large number of microbial proteins in a high-throughput and standardized manner. In yeast, pioneering studies have demonstrated that it is possible to generate expression clones of $85 \%-90 \%$ of the open reading frames for large-scale biochemical analysis (23). More recently, the entire proteome of a yeast strain has been ar- 
rayed on glass slides and screened for binding of proteins and lipids (38). Novel cloning and expression procedures have the potential to overcome the difficulties of large scale traditional cloning. One of these systems, the Gateway cloning system, is increasingly being used to clone large numbers of coding sequences from sequenced genomes (34). PCR products of each coding sequence are mass-generated using specific oligonu- cleotides that incorporate bacteriophage recombination sites. In the presence of the appropriate recombinase, the amplified genes are inserted in the vector and screened. The empty vector contains a suicide gene that permits growth only in a limited number of bacterial strains. Nonrecombinant clones fail to propagate. Once the gene has been inserted into an entry vector, it can be shuffled to any number of different expression vectors for production in bacterial, mammalian, or insect cell lines. The protein can also have different types of tags, depending on the vector used, and these tags are useful during the purification of the protein from cells. All the reactions involved are simple and rapid in contrast to traditional digestion and cloning procedures. This strategy will allow the development of large expression banks for the selected microbial proteins. Individual clones will be identified within the bank using insert-specific probes hybridizing either onto the coding sequence or onto unique noncoding flag sequences inserted with the amplification procedure.

We are currently developing protein microarrays containing malaria parasite proteins that, on the basis of their primary sequence or from experimental evidence, are either secreted or localized on the surface of malaria parasites. The first protein chip will contain most of the $P$. falciparum antigens currently being investigated for their ability to elicit protective immunity in animals and humans in vaccine trials. A second generation of microarrays will contain recombinant proteins that, on the basis of their primary structure or from experimental evidence, are predicted to be either secreted or exposed on the surface of the malaria parasite. These molecules are likely to be directly exposed to the action of serum antibodies and, therefore, could function as targets of natural acquired immunity. The performance of the microarrays in terms of sensitivity, reproducibility and stability will be validated using a group of serum samples that have been collected from individuals living in different areas of a malaria endemic country (28). Preliminary results indicate that the immune response detected by protein microarrays against a set of arrayed malaria antigens is in agreement with previous data obtained by analyzing the same group of sera in ELISA and immunoblot. Protein microarrays, containing a significant fraction of the bacterial and parasitic proteome, will shed new light on both the quantitative and qualitative aspects of the humoral immune response. The development of protein microarrays for the analysis of the cellmediated immune response remains a major challenge. In the near future, hydrogel-coated slides will facilitate the development of cell arrays. In combination with cell arrays, progress

Figure 4. Schematic and fluorescent scans of the arrays employed for the clinical diagnosis of allergies. (A) Schematic representation of the array. Colored circles indicate the positions where the allergens were printed in quadruplicate and the internal $\mathrm{IgE}$ calibration curve was printed in duplicate. The blank (dilution buffer) is represented as white circles. (B) Fluorescent scan of allergen arrays incubated with two different serum samples and developed using the tyramide amplification protocol. 
in both antigen arraying and in assays for cytokine detection at the site of production, promises to overcome current limitations to our understanding of the cell-mediated immune response.

\section{ACKNOWLEDGMENTS}

We would like to acknowledge the excellent scientific and technical contributions of our colleagues, especially Letizia Mezzasoma, Andrea Ardizzoni, and Julian Gray.

\section{REFERENCES}

1.Bacarese-Hamilton, T., L. Mezzasoma, C. Ingham, A. Ardizzoni, R. Rossi, F. Bistoni, and A. Crisanti. 2002. Detection of allergen-specific IgE on microarrays by use of signal amplification techniques. Clin. Chem. 48:13671370 .

2.Baird, J.K. 1995. Host age as a determinant of acquired immunity to Plas modium falciparum. Parasitol. Today 11:105-111.

3.Ben Mamoun, C., I.Y. Gluzman, C. Hott, S.K. MacMillan, A.S. Amarakone, D.L. Anderson, J.M. Carlton, J.B. Dame, et al. 2001. Co-ordinated programme of gene expression during asexual intraerythrocytic development of the human malaria parasite Plasmodium falciparum revealed by microarray analysis. Mol. Microbiol. 39:26-36.

4.Bojang, K.A., P.J. Milligan, M. Pinder, L. Vigneron, A. Alloueche, K.E Kester, W.R. Ballou, D.J. Conway, et al. 2001. Efficacy of RTS,S/AS02 malaria vaccine against Plasmodium falciparum infection in semi-immune adult men in The Gambia: a randomised trial. Lancet 358:1927-1934.

5.Bussow, K., D. Cahill, W. Nietfeld, D. Bancroft, E. Scherzinger, H. Lehrach, and G. Walter. 1998. A method for global protein expression and antibody screening on high-density filters of an arrayed cDNA library. Nucleic Acids Res. 26:5007-5008.

6.Bussow, K., E. Nordhoff, C. Lubbert, H. Lehrach, and G. Walter. 2000. A human cDNA library for high-throughput protein expression screening. Genomics 65:1-8.

7.Cahill, D.J. 2001. Protein and antibody arrays and their medical applications. J. Immunol. Methods 250:81-91.

8.Carucci, D.J. 2001. Genomic tools for gene and protein discovery in malaria: toward new vaccines. Vaccine 19:2315-2318.

9.de Wildt, R.M., C.R. Mundy, B.D. Gorick, and I.M. Tomlinson. 2000. Antibody arrays for high-throughput screening of antibody-antigen interactions. Nat. Biotechnol. 18:989-994.

10.Ekins, R.P. 1989. Multi-analyte immunoassay. J. Pharm. Biomed. Anal. 7:155-168.

11.Ekins, R., F. Chu, and E. Biggart. 1990. Multispot, multianalyte, immunoassay. Ann. Biol. Clin. (Paris) 48:655-666.

12.Emili, A.Q. and G. Cagney. 2000. Large-scale functional analysis using peptide or protein arrays. Nat. Biotechnol. 18:393-397.

13.Engers, H.D. and T. Godal. 1998. Malaria vaccine development: current status. Parasitol. Today 14:56-64.

14.Ge, H. 2000. UPA, a universal protein array system for quantitative detection of protein-protein, protein-DNA, protein-RNA and protein-ligand interactions. Nucleic Acids Res. 28:e3.

15.Haab, B.B., M.J. Dunham, and P.O. Brown. 2001. Protein microarrays for highly parallel detection and quantitation of specific proteins and antibodies in complex solutions. Genome Biol. 2:1-13.

16.Hayward, R.E., J.L. Derisi, S. Alfadhli, D.C. Kaslow, P.O. Brown, and P.K. Rathod. 2000. Shotgun DNA microarrays and stage-specific gene expression in Plasmodium falciparum malaria. Mol. Microbiol. 35:6-14.

17.Huang, R.P., R. Huang, Y. Fan, and Y. Lin. 2001. Simultaneous detection of multiple cytokines from conditioned media and patient's sera by an antibody-based protein array system. Anal. Biochem. 294:55-62.

18.Joos, T.O., M. Schrenk, P. Hopfl, K. Kroger, U. Chowdhury, D. Stoll, D. Schorner, M. Durr, et al. 2000. A microarray enzyme-linked immunosorbent assay for autoimmune diagnostics. Electrophoresis 21:2641-2650.

19.Kricka, L. 1993. Trends in immunoassay technologies. J. Clin. Immunoassay 16:267-271

20.Lorenz, M. 2002. Genomic approaches to fungal pathogenicity. Curr. Opin. Microbiol. 5:372-378
21.Lueking, A., M. Horn, H. Eickhoff, K. Bussow, H. Lehrach, and G. Walter. 1999. Protein microarrays for gene expression and antibody screening. Anal. Biochem. 270:103-111.

22.MacBeath, G. and S.L. Schreiber. 2000. Printing proteins as microarrays for high-throughput function determination. Science 289:1760-1763.

23.Martzen, M.R., S.M. McCraith, S.L. Spinelli, F.M. Torres, S. Fields, E.J. Grayhack, and E.M. Phizicky. 1999. A biochemical genomics approach for identifying genes by the activity of their products. Science 286:1153-1155.

24.Mezzasoma, L., T. Bacarese-Hamilton, M. Di Cristina, R. Rossi, F. Bistoni, and A. Crisanti. 2002. Antigen microarrays for serodiagnosis of infectious diseases. Clin. Chem. 48:121-130.

25.Mills, J., K. Roth, R. Cagan, and J. Gordon. 2001. DNA microarrays and beyond: completing the journey from tissue to cell. Nat. Cell Biol. 3:E175E178.

26.Molecular Probes, Inc. 2001. Tyramide Signal Amplification Kits. Product Information MP20911. Molecular Probes, Inc., Eugene, OR.

27.Richie, T.L. and A. Saul. 2002. Progress and challenges for malaria vaccines. Nature 415:694-701.

28.Robson, K.J., A. Dolo, I.R. Hackford, O. Doumbo, M.B. Richards, M.M. Keita, T. Sidibe, A. Bosman, et al. 1998. Natural polymorphism in the thrombospondin-related adhesive protein of Plasmodium falciparum. Am. J. Trop. Med. Hyg. 58:81-89.

29.Schena, M., R.A. Heller, T.P. Theriault, K. Konrad, E. Lachenmeier, and R.W. Davis. 1998. Microarrays: biotechnology's discovery platform for functional genomics. Trends Biotechnol. 16:301-306.

30.Schoolnik, G.K. 2002. Microarray analysis of bacterial pathogenicity. Adv. Microb. Physiol. 46:1-45.

31.Schweitzer, B., S. Wiltshire, J. Lambert, S. O’Malley, K. Kukanskis, Z. Zhu, S.F. Kingsmore, P.M. Lizardi, et al. 2000. Inaugural article: immunoassays with rolling circle DNA amplification: a versatile platform for ultrasensitive antigen detection. Proc. Natl. Acad. Sci. USA 97:1011310119.

32.Silzel, J.W., B. Cercek, C. Dodson, T. Tsay, and R.J. Obremski. 1998. Mass-sensing, multianalyte microarray immunoassay with imaging detection. Clin. Chem. 44:2036-2043.

33.van Gijlswijk, R.P., H.J. Zijlmans, J. Wiegant, M.N. Bobrow, T.J. Erickson, K.E. Adler, H.J.Tanke, and A.K. Raap. 1997. Fluorochrome-labeled tyramides: use in immunocytochemistry and fluorescence in situ hybridization. J. Histochem. Cytochem. 45:375-382.

34.Walhout, A.J., G.F. Temple, M.A. Brasch, J.L. Hartley, M.A. Lorson, S. van den Heuvel, and M. Vidal. 2000. GATEWAY recombinational cloning: application to the cloning of large numbers of open reading frames or ORFeomes. Methods Enzymol. 328:575-592.

35.Walter, G., K. Bussow, D.J. Cahill, A. Lueking, and H. Lehrach. 2000. Protein arrays for gene expression and molecular interaction screening. Curr. Opin. Microbiol. 3:298-302.

36.Wiese, R., Y. Belosludtsev, T. Powdrill, P. Thompson, and M. Hogan. 2001. Simultaneous multianalyte ELISA performed on a microarray platform. Clin. Chem. 47:1451-1457.

37.Wiltshire, S., S. O’Malley, J. Lambert, K. Kukanskis, D. Edgar, S.F. Kingsmore, and B. Schweitzer. 2000. Detection of multiple allergen-specific IgEs on microarrays by immunoassay with rolling circle amplification. Clin. Chem. 46:1990-1993.

38.Zhu, H., M. Bilgin, R. Bangham, D. Hall, A. Casamayor, P. Bertone, N. Lan, R. Jansen, et al. 2001. Global analysis of protein activities using proteome chips. Science 293:2101-2105.

39.Zhu, H., J.F. Klemic, S. Chang, P. Bertone, A. Casamayor, K.G. Klemic, D. Smith, M. Gerstein, et al. 2000. Analysis of yeast protein kinases using protein chips. Nat. Genet. 26:283-289.

\section{Address correspondence to:}

Dr. Andrea Crisanti

Department of Biology

Imperial College of Science, Technology and Medicine

Imperial College Road

London SW7 2AZ, UK

e-mail:acrs@ic.ac.uk 